\title{
BioéthiqueOnline
}

\section{Un jeu dont vous êtes le héros : « Entre idéal et réalité : en Jeu(x) éthique(s) de la recherche "}

\section{Émilie Peter, Claire Dupont, Thérèse Yéro Adamou et Maria Ruiz-Castell}

Volume 4, 2015

Reçu : 8 Mar 2014; publié : 16 Jul 2015; éditeurs : Bryn Williams-Jones \& Maude Laliberté

URI : https://id.erudit.org/iderudit/1035506ar

DOI : https://doi.org/10.7202/1035506ar

Aller au sommaire du numéro

Éditeur(s)

BioéthiqueOnline

ISSN

1923-2799 (numérique)

Découvrir la revue

Citer cet article

Peter, É., Dupont, C., Adamou, T. Y. \& Ruiz-Castell, M. (2015). Un jeu dont vous êtes le héros : « Entre idéal et réalité : en Jeu(x) éthique(s) de la recherche ». BioéthiqueOnline, 4. https://doi.org/10.7202/1035506ar
Résumé de l'article

Le principe de ce jeu, qui s'adresse essentiellement aux jeunes chercheurs, est le suivant : les participants sont invités à incarner une équipe de recherche, chargée d'étudier les impacts sanitaires inhérents à la conduite d'activités minières en Bolivie. Ce jeu interactif, qui promeut la réflexion éthique et la prise de décision par un processus dialogique et réflexif, a été conçu afin de mettre en pratique l'intégration de la prise de décision, l'interaction entre les participants et la construction conjointe de sens.
Droits d'auteur (C) E Peter, C Dupont, T Y Adamou et M Ruiz-Castell, 2015

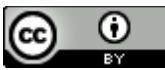

Ce document est protégé par la loi sur le droit d'auteur. L'utilisation des services d'Érudit (y compris la reproduction) est assujettie à sa politique d'utilisation que vous pouvez consulter en ligne.

https://apropos.erudit.org/fr/usagers/politique-dutilisation/ 


\title{
Un jeu dont vous êtes le héros : "Entre idéal et réalité : en $\operatorname{Jeu}(x)$ éthique(s) de la recherche »
}

\author{
COMMENTAIRE / COMMENTARY \\ Émilie Peter ${ }^{1}$, Claire Dupont ${ }^{2}$, Thérèse Yéro Adamou ${ }^{3}$ Maria Ruiz-Castell $^{2}$ \\ Reçu/Received: 8 Mar 2014 \\ Publié/Published: 16 Jul 2015 \\ Éditeurs/Editors: Bryn Williams-Jones \& Maude Laliberté \\ 2015 E Peter, C Dupont, T Y Adamou \& M Ruiz-Castell, Creative Commons Attribution 4.0 International License
}

\section{Résumé}

Le principe de ce jeu, qui s'adresse essentiellement aux jeunes chercheurs, est le suivant: les participants sont invités à incarner une équipe de recherche, chargée d'étudier les impacts sanitaires inhérents à la conduite d'activités minières en Bolivie. Ce jeu interactif, qui promeut la réflexion éthique et la prise de décision par un processus dialogique et réflexif, a été conçu afin de mettre en pratique l'intégration de la prise de décision, l'interaction entre les participants et la construction conjointe de sens.

\section{Mots clés}

bioéthique, enseignement, jeu interactif, prise de décision, éthique de la recherche

\section{Summary}

The principle of this game, which is primarily aimed at junior researchers, is as follows: participants are asked to embody a research team studying the health impacts associated with the conduct of mining activities in Bolivia. This interactive game, which promotes ethical reflection and decision-making through a dialogical and reflexive process, was designed to put into practice the integration of decision-making, interaction between the participants and the co-construction of meaning.

\section{Keywords}

bioethics, teaching, interactive game, decision making, research ethics

\section{Affiliations des auteurs / Author Affiliations}

${ }^{1}$ École nationale d'administration publique (ÉNAP), Montréal, Canada

${ }^{2}$ Centre de recherche du CHU de Québec et Département de médecine sociale et préventive, Faculté de médecine, Université Laval, Québec, Canada

${ }^{3}$ Faculté des sciences infirmières, Université Laval, Québec, Canada

\section{Correspondance / Correspondence}

Émilie Peter, emilie.peter@enap.ca

\section{Remerciements}

Les auteurs souhaitent remercier pour leur aide apportée à ce projet : les chercheurs de l'étude, à partir de laquelle le scénario du jeu a été inspiré, sans leur accord et confiance le jeu n'aurait pas pu être développé; le professeur Bernard Roy de la Faculté des sciences infirmières (Université Laval) pour sa confiance et son soutien au projet; la professeure Florence Piron du Département d'information et de communication (Université Laval) pour ses conseils; l'équipe de recherche du Centre de recherche du $\mathrm{CHU}$ de Québec pour avoir accepté de participer à un pré-test de l'activité; ainsi que toutes les personnes qui ont accepté de participer aux activités en tant que complices, pour leurs encouragements et disponibilités.

\section{Conflit d'intérêts}

Aucun déclaré

\section{Acknowledgements}

The authors would like to thank the following people for the help with this project: researchers involved in the study from which the game's storyline was inspired, without whose support and trust the game could not have been developed; Professor Bernard Roy, Faculty of Nursing (Université Laval) for his encouragement and support for the project; Professor Florence Piron, Department of Information and Communication (Université Laval) for her advice; the research team of the Research Centre of the University Hospital of Quebec for agreeing to participate in a pre-test of the game; and all those who agreed to participate in the game, for their encouragement and availability.

\section{Conflicts of Interest}

None declared 


\section{Préambule}

Le jeu interactif décrit dans ce texte est sous forme de PowerPoint - diaporama (11Mg), PPTX $(11 \mathrm{Mg})$, guide d'animation $(9 \mathrm{Mg})$ - et associé à un vidéo (en français, et avec sous-titres anglais) afin d'illustrer le concept de l'activité. Toutes les modifications ou améliorations au jeu sont autorisées après contact avec l'un des auteurs. Le jeu est destiné à une utilisation à titre individuel ou collectif, pour toute personne qui souhaiterait s'exercer sur les concepts éthiques ou dans le cadre d'une activité de formation. L'objectif est de faciliter la diffusion et l'utilisation de l'activité sans la présence d'animateurs spécifiques, grâce à l'inclusion d'encarts de textes de réflexion pour le/les joueur(s). Idéalement des vidéos seront développés afin de remplacer les animateurs.

\section{Introduction}

En raison de la préoccupation grandissante envers le renforcement des comportements éthiques des praticiens et des chercheurs, l'enseignement de l'éthique se trouve au centre de multiples débats. Malgré la remise en question de sa pertinence par le passé, de nombreuses voix s'élèvent aujourd'hui pour souligner la nécessité de l'enseignement de l'éthique aux futurs professionnels concernés [1-4]. Celui-ci peut avoir plusieurs objectifs, notamment pour viser à : accroître la sensibilité éthique des participants, accroître leur connaissance, améliorer leur jugement éthique ou encore influencer leur personnalité éthique [2].

Pour atteindre ces objectifs, plusieurs méthodes pédagogiques existent, leur valeur étant fonction de la finalité et du contexte d'apprentissage [1]. Toutefois, l'acquisition de compétences concernant le raisonnement et la prise de décision s'avère de plus en plus valorisée comparativement à l'enseignement des théories éthiques [5,6]. En effet, l'impact du seul enseignement théorique en salle de cours a été remis en question [2] et plusieurs auteurs incitent aujourd'hui à la prudence face à ce type d'enseignement, compte tenu des risques potentiels d'endoctrinement qu'il comporte [2,3,7]. De plus, les codes éthiques ne pouvant prévoir et donc parer à toutes situations, la formation axée sur l'éthique dans la pratique est de plus en plus plébiscitée [8]. Un mouvement laissant une plus large place à la discussion, l'interaction [2,4,7], la contextualisation de la réflexion, ou encore à la construction conjointe de sens s'est ainsi développé [9]. Enfin, certains auteurs remettent également en question la pertinence des cours formels, arguant que l'instruction de l'éthique à elle seule ne peut mener à l'adoption d'un comportement éthique. D'après ces derniers, il est indispensable d'intégrer cet apprentissage dans le curriculum « informel » [10] ou "caché » [11], c'est-à-dire, intégré au quotidien dans le contexte de pratique. Les controverses restent donc toujours vives quant à l'objectif et la méthode d'enseignement de l'éthique à des praticiens et des chercheurs [2].

Prenant part à ces discussions, nous souhaitons présenter une activité pédagogique développée en 2013 dans le cadre du colloque Enjeux de la recherche organisé par l'Association francophone pour le savoir (ACFAS). Notre constat de la difficulté pour les chercheurs d'intégrer les multiples dimensions de l'éthique dans le contexte de pratique, et ce, malgré les différentes formations en éthique, a été l'origine du développement du jeu. En s'inscrivant dans une réflexion sur l'éthique des codes et l'éthique de la pratique [12], nous avons cherché à accentuer la dimension contextuelle de la réflexion éthique en sensibilisant aux difficultés d'application des normes et règlements en éthique de la recherche au Canada.

Nous discuterons tout d'abord des objectifs pédagogiques du jeu, ainsi que de sa pertinence en lien avec notre positionnement en faveur des méthodes pédagogiques contextuelles, dialogiques et interactives. Nous décrirons ensuite son support, ses mécanismes et ses règles. Enfin, nous conclurons par la contribution et les perspectives de développement envisagées pour cette activité.

Partout à travers le monde le respect des principes éthiques en recherche apparaît comme un enjeu majeur. C'est d'ailleurs ce qui a mené le Groupe consultatif interagences en éthique de la recherche 
au Canada à renouveler l'Énoncé de politique des trois Conseils : Éthique de la recherche avec des êtres humains (EPTC2) [13]. L'activité pédagogique ici présentée doit ainsi être perçue comme une contribution additionnelle aux autres guides et outils éthiques développés, notamment par le Groupe consultatif (par exemple, les didacticiels et webinaires), en vue de favoriser la mise en pratique de principes éthiques.

\section{Les objectifs pédagogiques du jeu}

Le jeu a été développé afin de répondre aux trois objectifs pédagogiques suivants :

1. Faciliter la compréhension et l'intégration des principes éthiques en recherche afin d'en améliorer leur apprentissage.

2. Sensibiliser les chercheurs et les étudiants-chercheurs des cycles supérieurs aux enjeux relatifs à la mise en application des principes éthiques, tels que :

- la difficulté, une fois confronté à la réalité du terrain, de concilier les différentes contraintes rencontrées (éthiques ou autres);

- l'influence des a priori et des émotions dans la prise de décisions;

- la difficulté de prendre des décisions avec une connaissance (inévitablement) partielle du contexte.

3. Développer chez les chercheurs et étudiants-chercheurs certaines aptitudes de reconnaissance et d'anticipation envers les questionnements éthiques associés à la réalisation d'un projet de recherche, ainsi qu'envers les conséquences des réponses adoptées.

\section{Publics cibles}

Le jeu cible principalement un public de chercheurs et étudiants-chercheurs, mais aussi les praticiens dans des domaines connexes à la recherche (par exemple, l'évaluation).

\section{Ancrage dans les théories pédagogiques}

Le jeu a été développé autour de grands enjeux éthiques, qu'il nous semblait important de prendre en considération dans un contexte de pratique. En effet, tout travail de recherche s'inscrit dans un environnement social et politique particulier, impliquant un processus non linéaire et une évolution constante des identités et rôles de chacun [14]. Plusieurs auteurs ont ainsi souligné l'importance de l'acquisition d'habiletés particulières, au-delà de la connaissance des principes éthiques, afin de pouvoir répondre aux enjeux éthiques dans la pratique. Parmi ces habiletés, nous pouvons citer : la capacité à discerner les enjeux politiques; à imaginer les conséquences de ses actions; à reconnaître l'inévitable partialité de ces dernières; à être authentique quant à ses décisions [15]; à reconnaître les différences de pouvoirs, par ailleurs évolutives, entre chercheurs et participants, et à adapter son comportement en conséquence [16]. De plus, il est attendu des chercheurs qu'ils fassent preuve de flexibilité et d'empathie afin d'adapter les procédures éthiques au contexte de recherche (et non l'inverse) $[17,18]$. Inscrite dans la pratique, leur démarche éthique doit en effet leur permettre de se réapproprier l'éthique hors des préjugés et des technicités procédurales $[8,14,15,19]$ et prendre en compte les différentes dimensions de l'interculturalité [19]. Ces approches sont d'autant plus nécessaires lorsque le chercheur adopte une perspective participative [18] ou ancrée dans une recherche-action [20]. Enfin, sont également considérées comme primordiales : la réflexivité continue des chercheurs vis-à-vis des enjeux éthiques [12] et leur questionnement personnel sur les définitions multiples de leur rôle et de la place de la recherche dans la société. 


\section{Buts poursuivis}

Le jeu a été élaboré en vue de souligner :

- la présence de nombreux types d'enjeux éthiques dans une recherche (les conflits d'intérêts, l'inclusion et l'exclusion des participants, l'accès et la distribution des risques et bénéfices, l'autonomie et le respect des participants, la responsabilité et l'implication locale du chercheur, etc.);

- la présence de questionnements éthiques tout au long du processus d'une recherche et l'impact des choix d'une étape à l'autre;

- l'importance d'analyser la valeur éthique des différentes possibilités d'action et de leurs répercussions potentielles sur l'ensemble des étapes subséquentes de la recherche, ainsi que sur les différents acteurs en présence. Cette analyse nécessite de tenir compte du fait que les principes interagissent les uns avec les autres; que leur application requiert une certaine flexibilité; ou encore, de considérer la multiplicité des cadres éthiques des différents acteurs;

- la difficulté de prendre une décision et d'analyser le contexte lorsque l'information accessible, comme dans toute recherche, est limitée (pour plus de réalisme, nous avons repris les connaissances initiales des chercheurs ayant réalisé la recherche), ainsi que;

- l'impact de l'implication émotionnelle et de la confrontation avec les positions morales personnelles dans le processus décisionnel.

Nous avons principalement basé notre démarche sur les lignes directrices en éthique de la recherche au Canada, soit l'EPTC2 [13]. Nous avons également pris en considération d'autres guides développés au Canada, s'adressant à des communautés particulières [21-25].

\section{La description du jeu}

Ce jeu interactif - qui utilise une plateforme PowerPoint comme support - est largement inspiré des " livres dont vous êtes le héros ". Dans ces livres, le lecteur est le maître de l'enchaînement des évènements et du déroulement de l'histoire. À chaque étape et en fonction de son choix, celui-ci est orienté vers le chapitre correspondant selon ses envies et préférences, influençant ainsi le déroulement et l'issue de l'histoire. Ce format vise ainsi à favoriser la réflexivité des participants, principalement des jeunes chercheurs, face à leur perception de l'éthique et à son application dans un environnement complexe. Dans le cas présent, les participants sont invités à incarner une équipe de recherche qui va étudier les impacts d'activités minières sur la santé de la population en Bolivie (voir la présentation officielle du jeu dans l'Encadré 1). Confrontés au cours du jeu à toute une série d'enjeux éthiques, leur défi sera de réfléchir, discuter et décider ensemble de la stratégie à adopter pour mener à bien - et le plus éthiquement possible - leur projet. 


\section{Encadré 1 : Présentation officielle du jeu \\ Devenez pour le temps d'une partie un chercheur " éthiquement " hors pair!}

Connaissez-vous les jeux dont vous êtes le héros? Oui, Non ? Peu importe, bienvenue dans cet univers investi pour l'occasion et appliqué à la réflexion sur les enjeux éthiques de la recherche. Le principe est simple : mener à bien le projet de recherche qui vous sera confié à l'aide d'un scénario interactif et surmonter les difficultés du terrain en partageant vos questionnements éthiques. Le tout dans une ambiance ludique!

\section{L'histoire commence...}

On vous propose d'évaluer les impacts potentiels sur la santé des résidus provenant de l'exploitation de mines installées depuis plusieurs années dans une petite ville bolivienne au sud de la Paz. II s'agit de la 1ère étude de ce genre réalisée en Bolivie; vous saisissez donc l'opportunité sans hésiter. Plus précisément, vous allez devoir vous intéresser aux effets des métaux lourds rejetés dans l'environnement par ces mines, sur le développement des enfants de la ville.

\section{Constituez votre équipe de chercheurs et embarquez!}

Vous commencerez votre mission directement sur place où toute l'organisation du projet est à mettre en œuvre. Localement, la coopération vous paraît plutôt bonne, pourtant, rapidement, vous allez vous rendre compte que de nombreux obstacles éthiques tendent à compliquer vos plans. Pour y faire face, plusieurs stratégies possibles seront envisageables, alors comment allez-vous faire? Qu'allez-vous décider? Attention! Vos choix moduleront la suite des évènements et l'issue de l'histoire. II faudra donc faire preuve de discernement, de réflexivité, d'esprit critique et privilégier la collaboration afin d'opter pour l'option qui vous paraîtra la plus acceptable sur le plan éthique.

\section{Au cours de la partie...}

Heureusement, plusieurs personnes pourront vous venir en aide. Le chercheur qui a réellement accompli ce projet sera présent et pourra répondre à vos questions. Un conseiller en éthique pourra vous rappeler certains principes théoriques et les animateurs du jeu pourront vous apporter des informations complémentaires. Cependant, des évènements imprévisibles pourront aussi avoir un impact sur vos choix... II vous faudra rester vigilants!

\section{À vous de jouer!}

Un des aspects innovants du jeu est que le scénario a été développé d'après une recherche déjà réalisée. Ainsi, les enjeux intégrés dans l'exercice sont des questionnements éthiques auxquels les chercheurs ont eu à faire face, ou qui auraient pu se poser dans le contexte de leur recherche. Cet aspect nous a semblé indispensable afin de développer un scénario qui permette de créer ce lien étroit entre sensibilisation à l'éthique, pratique de recherche et réalité du terrain. Prenant la forme d'un atelier collectif, le jeu implique initialement la présence d'au moins trois animateurs, à savoir: un présentateur qui explique et anime le jeu, notamment en encourageant les débats et la présentation des arguments de chacun; un conseiller en éthique qui souligne les enjeux éthiques abordés afin d'alimenter la discussion et répond aux questions des participants à ce sujet; et un animateurchercheur qui présente la recherche et répond aux questions des participants concernant le contexte du projet (dans le cas de futures animations du jeu, ces fonctions peuvent être cumulées par un même animateur).

Afin d'alimenter et de renforcer la discussion, il est également possible d'inclure des complices incognito parmi les participants. Leur rôle sera alors de tenter d'influencer la discussion et de susciter le débat (ex.: un complice défend les intérêts des acteurs politiques locaux, un autre les préoccupations de l'hôpital, un autre représente la voix de la population, un autre celle des compagnies minières, etc.). Cependant la présence de ces complices n'est qu'optionnelle. 
Le jeu débute par la présentation du projet et du contexte de recherche par l'animateur-chercheur. Après cette brève introduction, les participants sont dès le départ confrontés à un premier dilemme éthique. Trois options leur sont présentées pour faire face au problème (voir exemple Figure 1). Les participants sont alors invités à lire les réponses proposées, poser des questions et à voter individuellement. Les réponses des participants sont alors collectées en temps réel grâce à des télévoteurs individuels et à l'utilisation du logiciel CPS pour PowerPoint. Les résultats, une fois compilés, sont ensuite affichés à l'écran. Une discussion peut être engagée autour des différents choix, positions et arguments respectifs de chacun.

\section{Comment trouver du financement pour la ville de Potosi?}

Vous décidez:

A. De faire appel aux compagnies minières pour obtenir un budget supplémentaire ...

B. De ne réaliser l'étude qu'à Oruro, mais de cibler tous les sites contaminés...

$\checkmark$ C. De n'exclure aucune ville et d'aller chercher des fonds supplémentaires sans faire appel aux compagnies minières $\underline{-\cdots}$

Figure 1. Exemple de diapositive PowerPoint avec des questions à choix multiples

Le choix qui a obtenu la majorité des votes est retenu et va conditionner la suite des évènements (en cas d'égalité, une nouvelle discussion peut être proposée suivie d'un second vote). En fonction de leur choix, les participants sont amenés vers un autre questionnement " éthique " (avec de nouveaux enjeux). Une liste d'options leur est alors de nouveau proposée et ainsi de suite...

À chaque enjeu abordé, les animateurs auront au préalable pris le soin de souligner oralement que tous les choix présentés peuvent entraîner des conséquences à la fois positives et négatives en fonction du contexte (quant au processus et/ou à la finalité de l'action), et qu'il n'y a donc ni bonnes, ni mauvaises réponses. Les questions à choix multiples ont ainsi été élaborées de manière à ce que les participants réalisent à travers leur expérience, qu'opter pour un choix à première vue éthique n'est pas forcément un gage de réussite et qu'il peut au contraire, engendrer d'autres dilemmes éthiques (voir exemple en Encadré 2).

\section{Encadré 2 : Exemple de dilemme éthique}

À titre d'exemple, à la Figure 1, plusieurs actions sont proposées. Les participants sont invités à se questionner sur le problème amené par la nécessité d'un financement complémentaire au projet initial. Grâce à ce dernier, il serait alors possible d'inclure dans la recherche un groupe de population plus large avec lequel l'étude serait vraisemblablement plus pertinente. Parmi les options possibles, le choix de travailler avec des entreprises locales à forts intérêts socio-économiques (comme les compagnies minières) peut être a priori perçu par certains comme " moins » éthique. Toutefois, ce choix entraine plusieurs types de conséquences dont les répercussions peuvent être identifiées comme négatives, tel que la potentialité des conflits d'intérêts générés dans ce cas, mais aussi comme positives, telle que l'inclusion potentielle d'un nombre plus important de bénéficiaires grâce au financement supplémentaire.

Tout au long du jeu, les participants auront à réfléchir et discuter autour de plusieurs grandes thématiques éthiques: les conflits d'intérêts et les partenariats, l'inclusion et l'exclusion des participants, l'accès et la distribution des risques et bénéfices, le respect et l'autonomie des participants, la responsabilité et l'implication locale du chercheur. Ces dernières sont agencées autour des étapes potentielles d'une recherche, à savoir: l'initiation de la recherche et la sélection des partenaires locaux, la sélection du lieu de l'étude et des participants, la recherche de financement, et le processus de collecte des données. 


\section{Vous arrivez sur place, votre mission commence...}

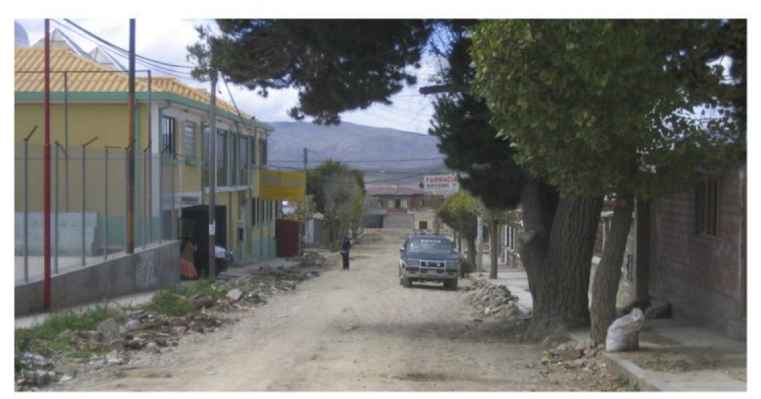

Figure 2. Exemple de diapositive PowerPoint avec mise en scène ludique
À l'issue de la partie, le chemin parcouru est discuté au regard des différents concepts éthiques abordés et à la lumière de la véritable " histoire " et des contraintes rencontrées par le chercheur qui a entrepris l'étude. En effet, selon les choix effectués par les participants, il est possible d'aboutir à un total de 20 scénarios différents.

La réalisation de l'activité nécessite la mise en place d'une atmosphère ludique (pour exemple, voir la Figure 2) et inspirant confiance afin d'encourager la fréquence, la spontanéité et l'authenticité des échanges entre participants.

\section{Conclusion}

En favorisant la réflexion, la prise de décision conjointe et en apportant du « réalisme " à l'apprentissage, ce jeu vise à renforcer le lien entre éthique, pratique de recherche et réalités du terrain. Il a notamment été conçu afin de rendre compte de la complexité inhérente à l'application de l'éthique, et ce dans le cadre d'une activité pédagogique en éthique de la recherche. Ainsi, plusieurs dimensions sont soulignées comme: la présence de plusieurs variables contextuelles et leur évolution au cours de la réalisation du projet; la multiplicité des positions "éthiques », impliquant toutes des conséquences positives et négatives; l'impact des décisions sur l'ensemble du processus de recherche; l'impact des repères culturels et disciplinaires dans la décision; ou encore, l'impact de l'implication émotionnelle et de la confrontation avec les positions morales personnelles dans le processus décisionnel. Par conséquent, cette activité se veut complémentaire aux guides éthiques et aux différentes activités de sensibilisation aux théories éthiques.

Le présent jeu a été testé à plusieurs reprises dans différents colloques et en cours d'éthique, lors : des activités Enjeux de la recherche au Congrès de l'ACFAS (le 9 mai 2013); d'un cours d'éthique intégré au Programme interuniversitaire de formation en recherche en santé mondiale, Santé-Cap (le 10 mai 2013); des rencontres Science et Société de Québec du Musée de la civilisation (le 28 novembre 2013); ainsi que de la Journée annuelle de l'enseignement de la Faculté de Médecine de l'Université Laval (le 9 avril 2014); ceci avec la présence d'animateurs et d'une des chercheurs ayant réalisé le projet. Enfin, le jeu a été conçu en vue de favoriser sa transférabilité à tout type de recherche. Ainsi, bien que la recherche présentée se déroule en Bolivie, cela ne restreint, ni la pertinence de la réflexion sur le processus cognitif de prise de décision en éthique, ni celle des principes éthiques abordés qui restent valides dans d'autres contextes de recherche.

\section{Voir le vidéo et à vous de jouer (diaporama) !}

(Nous invitons les joueurs à nous envoyer leurs commentaires ou nous aviser de tout problème rencontré lors de leur expérience, ce jeu étant en processus continu d'amélioration : claire.dupont27@gmail.com \& emilie.peter@enap.ca) 


\section{Références}

1. Callahan D. Goals in the Teaching of Ethics. In: Callahan D, Bok S, editors. Ethics Teaching in Higher Education. New York: Springer US; 1980. p. 61-80.

2. Davis M. Ethics and the University. London: Routledge; 1999.

3. Reiss MJ. Teaching Ethics in Science. Studies in Science Education. 1999;34(1):115-40.

4. Smith S, Fryer-Edwards K, Diekema DS, III CHB. Finding Effective Strategies for Teaching Ethics: A Comparison Trial of Two Interventions. Academic Medicine. 2004;79(3):265-71.

5. Lin CF, Lu MS, Chung CC, Yang CM. A comparison of problem-based learning and conventional teaching in nursing ethics education. Nursing Ethics. 2010;17(3):373-82.

6. Ubel PA. The Predictable Irrationality of Righteous Minds, and the Work of Ethicists. Hastings Center Report 43. 2013;3:18-22.

7. Downie R, Clarkeburn H. Approaches to the teaching of bioethics and professional ethics in undergraduate courses. Bioscience Education. 2005;5.

8. Guillemin M, Heggen K. The Narrative Approach as a Learning Strategy in the Formation of Novice Researchers. Qualitative Health Research. 2011;22(5):700-7.

9. Molewijk AC, Abma TA, Stolper M, Widdershoven GAM. Teaching ethics in the clinic. The theory and practice of moral case deliberation. Journal for Medical Ethics. 2008;34:120-4.

10. Hundert EM, Douglas-Steele D, Bickel J. Context in medical education: the informal ethics curriculum. Medical Education. 1996;30:353-64.

11. Hafferty FW, Franks R. The Hidden Curriculum, Ethics Teaching, and the Structure of Medical Education. Academic Medicine. 1994;69(11):861-71.

12. Guillemin M, Gillam L. Ethics, Reflexivity, and "Ethically Important Moments" in Research. Qualitative Inquiry. 2004;10(2):261-80.

13. Conseil de recherches en sciences humaines du Canada, Conseil de recherches en sciences naturelles et en génie du Canada, Instituts de recherche en santé du Canada. Énoncé de politique des trois Conseils : Éthique de la recherche avec des êtres humains. Ottawa: Groupe consultatif interagences en éthique de la recherche. 2010 Contract No.: MR21-18/2010F-PDF.

14. Halse C, Honey A. Rethinking Ethics Review as Institutional Discourse. Qualitative Inquiry. 2007;13(3):336-52.

15. Baarts C. Stuck in the middle: research ethics caught between science and politics. Qualitative Research. 2009;9(4):423-39.

16. Ben-Ari A, Enosh G. Power Relations and Reciprocity: Dialectics of Knowledge Construction. Qualitative Health Research. 2012;23(3):422-9.

17. Block K, Warr D, Gibbs L, Riggs E. Addressing Ethical and Methodological Challenges in Research with Refugee-background Young People: Reflections from the Field. Journal of Refugee Studies. 2012;26(1):69-87.

18. Boser S. Power, Ethics, and the IRB: Dissonance Over Human Participant Review of Participatory Research. Qualitative Inquiry. 2007;13(8):1060-74.

19. Tilley S, Gormley L. Canadian University Ethics Review: Cultural Complications Translating Principles Into Practice. Qualitative Inquiry. 2007;13(3):368-87.

20. Gelling L, Munn-Giddings C. Ethical Review of Action Research: The Challenges for Researchers and Research Ethics Committees. Research Ethics. 2011;7(3):100-6.

21. Association universitaire canadienne d'études nordiques (AUCEN). Principes d'éthique pour la conduite de la recherche dans le Nord. Ottawa: Association universitaire canadienne d'études nordiques. 2003.

22. Inuit Tapiriit Kanatami (ITK), Nunavut Research Institute (NRI). Negotiating Research Relationships with Inuit Communities. A Guide for Researchers. 2007.

23. Assemblée des Premières Nations du Québec et du Labrador. Protocole de recherche des Premières Nations du Québec et du Labrador. Wendake: Assemblée des Premières Nations du Québec et du Labrador. 2005. 
24. Inuit Tuttarvingat of NAHO, Inuit Tapiriit Kanatami. Inuit-Specific Perspectives on Research and Research Ethics. In: National Aboriginal Health Organization (NAHO), Inuit Tapiriit Kanatami (ITK) editors. 2010.

25. Centre des Premières Nations. Analyse et modèles d'éthique en recherche. Ottawa: Organisation nationale de la santé autochtone (ONSA). 2007. 